\title{
Atraumatic splenic ruptures triggered both remission and death in a single case of blastic plasmacytoid dendritic cell neoplasm
}

Keywords: Blastic plasmacytoid dendritic cell neoplasm, splenic rupture, dendritic cell, cancer vaccination, immunotherapy

\section{TO THE EDITOR}

A 78-year-old Japanese man presented to our hospital with multiple skin lesions, multiple lymphadenopathies and moderate splenomegaly (Figure 1). Hematological examination revealed a white blood cell (WBC) count of $13100 / \mu \mathrm{L}$ with $63 \%$ blasts, hemoglobin concentration of $12.2 \mathrm{~g} / \mathrm{dL}$ and platelet count of $42000 / \mu \mathrm{L}$. Blasts in the peripheral blood were medium in size and had scant grey-blue cytoplasm (Figure 2A). On flow cytometry analysis of peripheral blood, abnormal cells expressing CD2, CD4, CD7 and CD56 were observed. Based on these findings, we suspected leukemic transformation of lymphoma. Lymph node biopsy was performed and the specimen exhibited diffuse proliferation of large blastic cells (Figure 2B). These cells were positive for CD4, CD56, CD123 and TCL1 on immunohistochemistry (Figure 2C, D). Infiltration of these abnormal cells was also noted in the specimen from the skin lesion biopsy and bone marrow. The chromosomal analysis demonstrated a normal karyotype. Taken together, a diagnosis of blastic plasmacytoid dendritic cell neoplasm (BPDCN) was established. Fourteen days after the first visit, he returned with fever, anemia and tenderness in the left upper quadrant. We assumed progression of the disease and planned chemotherapy. Two days after admission, the WBC count, including blasts, started to decrease. Computed tomography (CT) demonstrated hematoma around the spleen and hemorrhagic ascites (Figure 3), indicating mild atraumatic splenic rupture (ASR). Serum chemistry revealed the elevation of C-reactive protein (CRP) and uric acid up to $18.58 \mathrm{mg} / \mathrm{dL}$ and $11.6 \mathrm{mg} / \mathrm{dL}$, respectively. These findings suggested that strong inflammation and tumor lysis occurred simultaneously after ASR. Chemotherapy was postponed and he was managed conservatively. Afterwards, he developed leukopenia together with slight improvement of skin lesions, but the mechanism of this phenomenon was unclear. His WBC count recovered gradually over 2 months, and blasts were finally undetectable in the peripheral blood. A slight reduction in abnormal blasts was noted in the bone marrow aspirate. Soon afterwards, blasts increased in the peripheral blood, and anemia, thrombocytopenia and skin lesions progressed. One month later, he was admitted again to receive chemotherapy. Three days before the scheduled date of chemotherapy, he developed fever and sudden pain in the left upper quadrant again. Subsequently, the WBC count began decreasing and hemorrhagic ascites was again detected on CT imaging (Figure 4A). The elevation of CRP and uric acid was also observed again. The skin lesions had visibly improved (Figure 4B) and the infiltration of abnormal blasts in the bone marrow markedly decreased. These findings
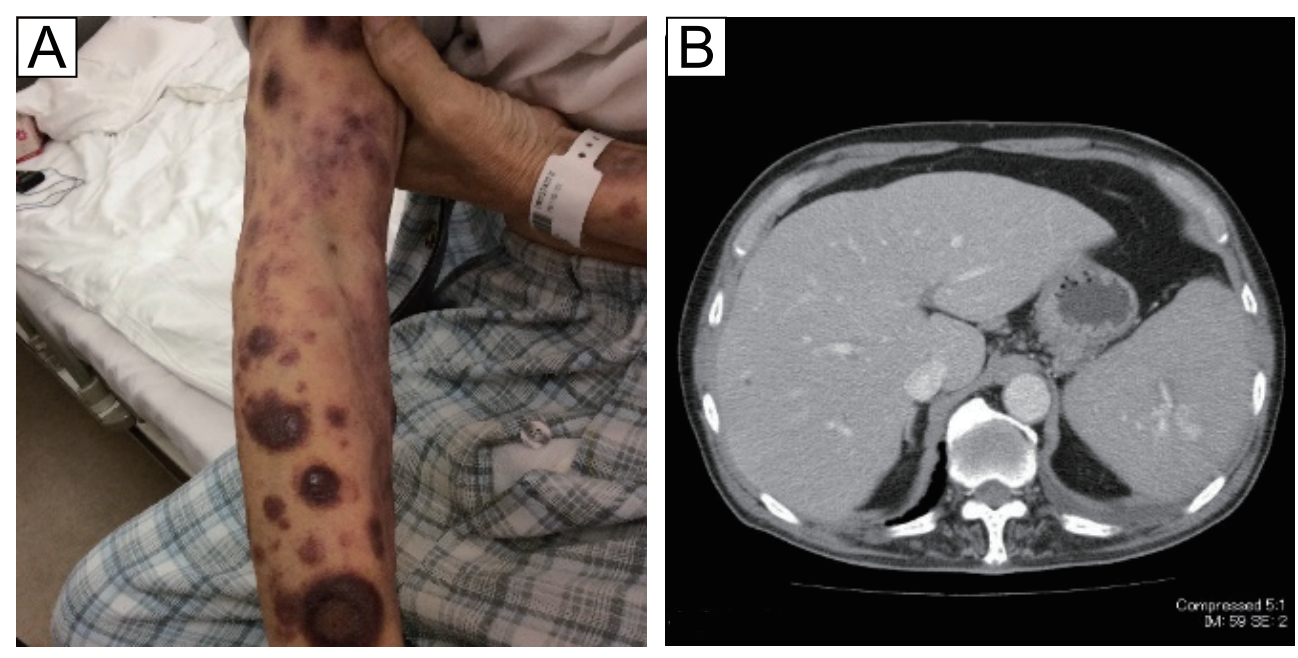

Fig. 1. The skin lesions and CT findings at the first visit. ( $\boldsymbol{A}$ ) Multiple dusky-red colored patches were systemically distributed. (B) Moderate splenomegaly was found on CT. CT, computed tomography. 

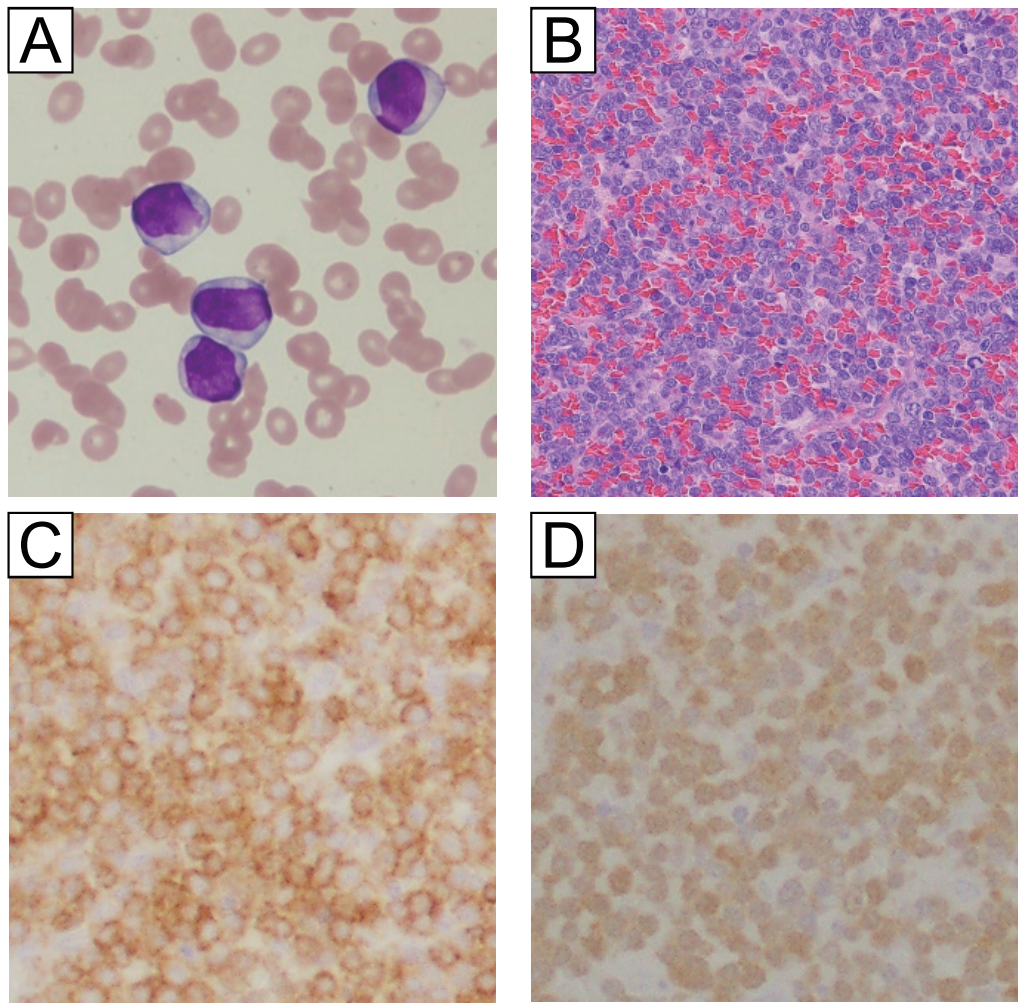

Fig. 2. Microscopic findings at the first visit. (A) Peripheral blood. Mediumsized blasts with scant grey-blue cytoplasm and fine chromatin were observed (B) Lymph node biopsy. Diffuse proliferation of large blastic cells was noted $(\boldsymbol{C}, \boldsymbol{D})$ Immunohistochemistry of the lymph node. $(\boldsymbol{C})$ Blastic cells were positive for CD123. (D) Blastic cells were positive for TCL1. TCL1, T cell leukemia/ lymphoma protein 1 .
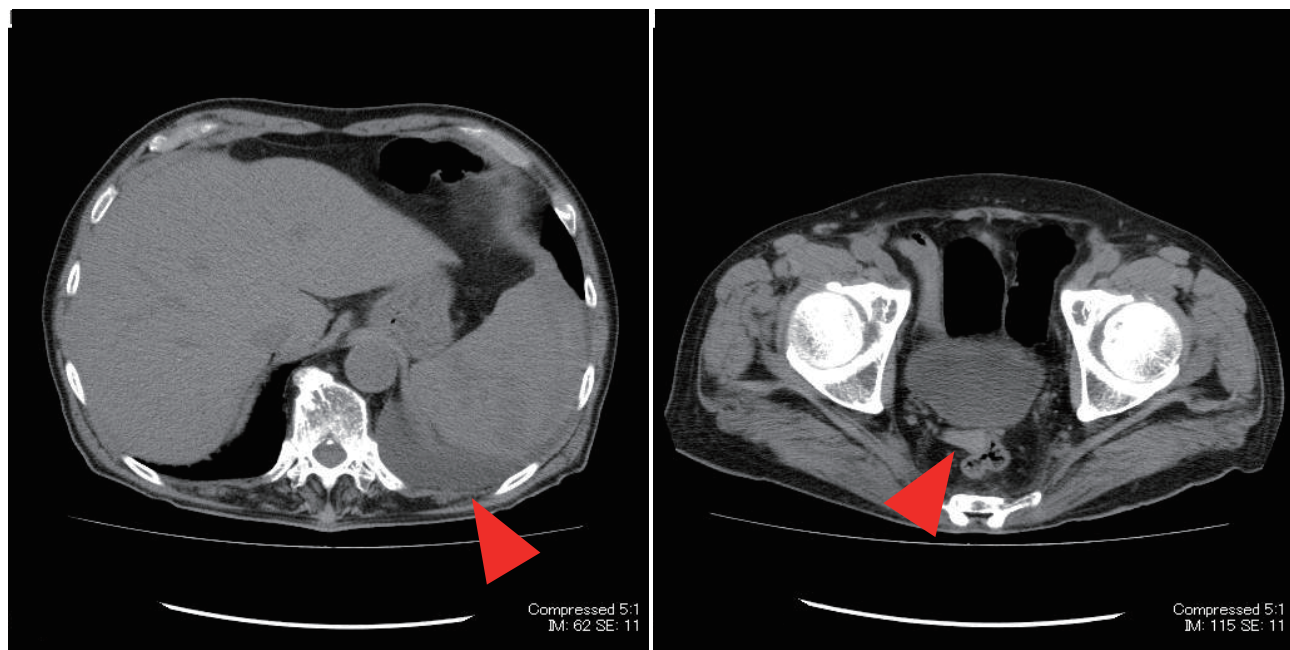

Fig. 3. CT findings at the first ASR. The red triangles indicate hematoma around the spleen (left panel) and hemorrhagic ascites (right panel). CT, computed tomography; ASR, atraumatic splenic rupture.

suggested that mild ASRs had repeatedly triggered the spontaneous partial remissions. Two months later, skin lesions started to progress, and thrombocytopenia appeared three months later. We considered disease progression, and started chemotherapy (THP-COP regimen consisting of cyclophosphamide, pirarubicin, vincristine and prednisolone, administered every three weeks). He exhibited immediate improvement of the blood cell count and skin lesions after the first chemotherapy cycle, but the disease gradually became resistant to the treatment during the eight cycles. He developed sudden hypovolemic shock soon after the eighth cycle of chemotherapy, and died within six hours. 


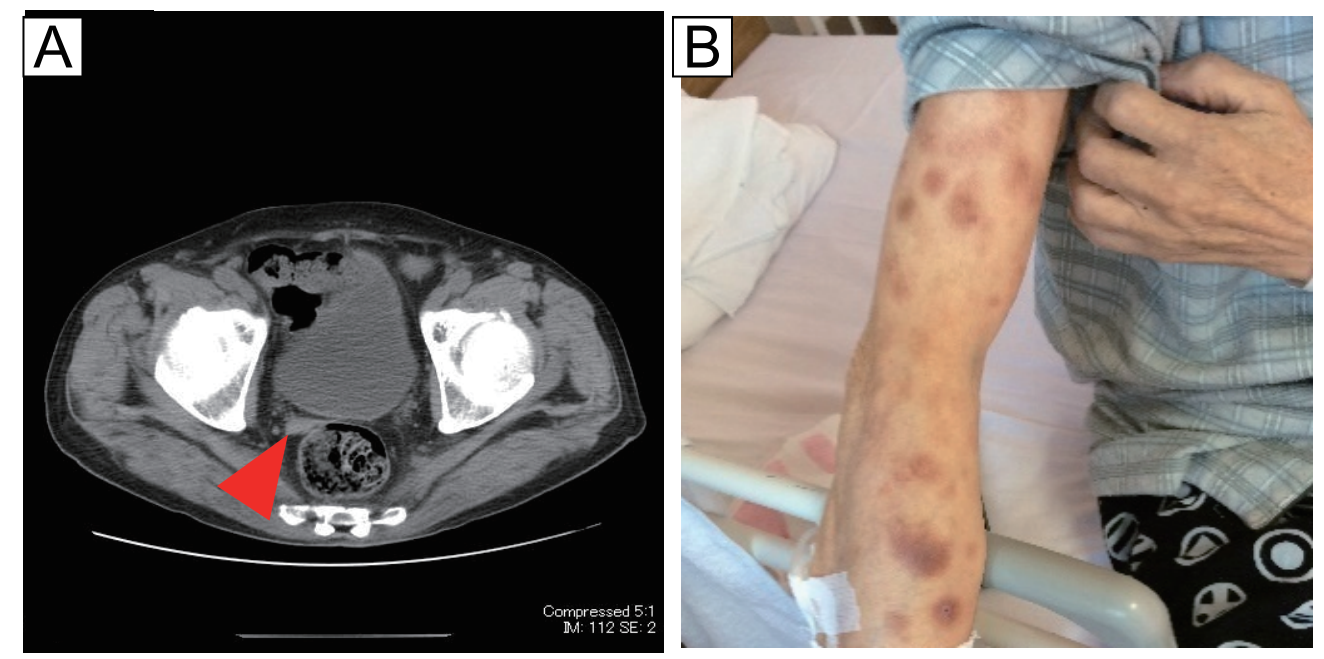

Fig. 4. ( $\boldsymbol{A})$ CT findings at the second ASR. Hemorrhagic ascites was found. (B) Skin lesions after the second ASR. Skin lesions improved after the ASR. CT, computed tomography; ASR, atraumatic splenic rupture.

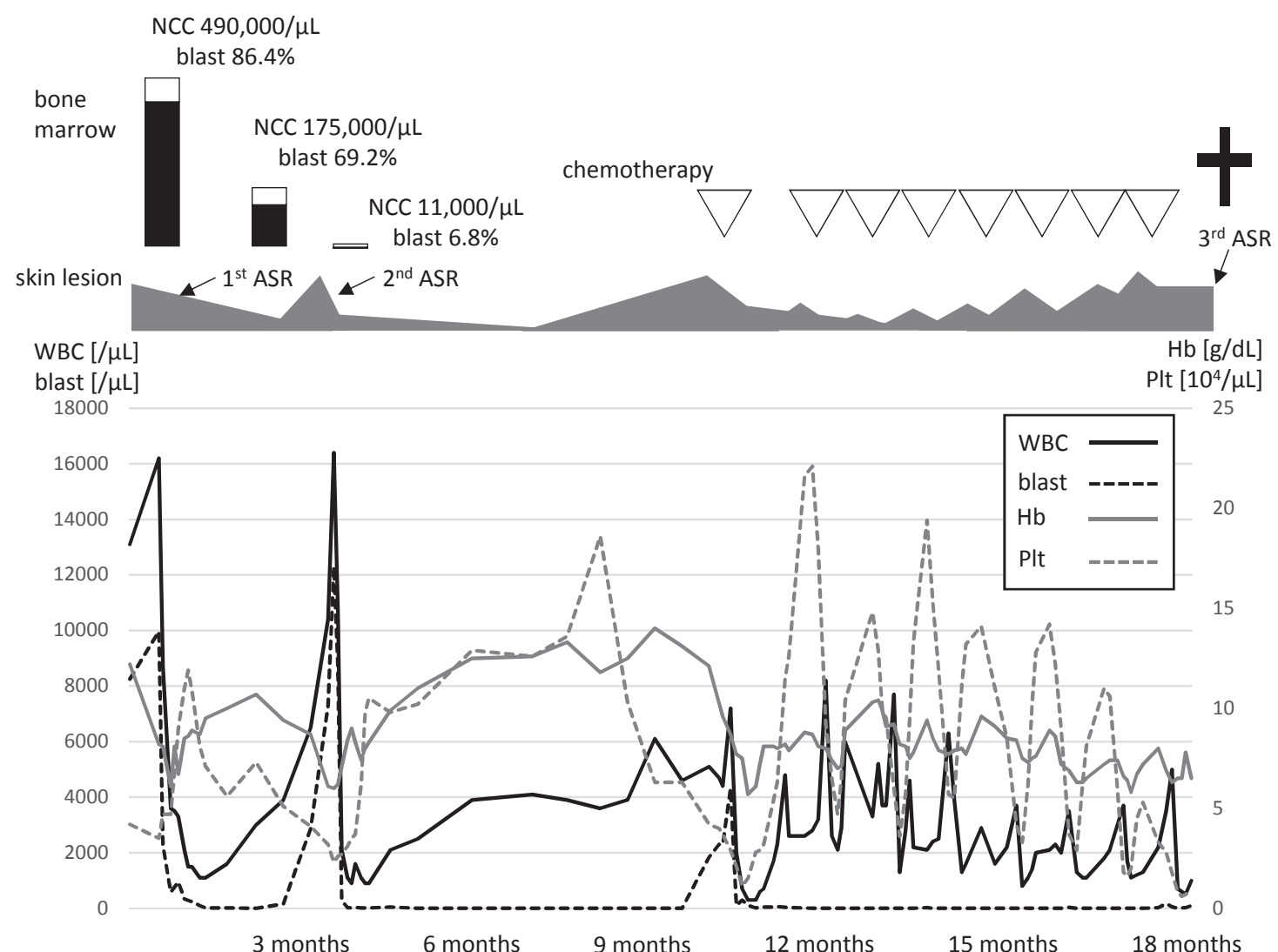

Fig. 5. Summary of the clinical course. The horizontal axis represents the time from the first visit. The cell counts in the serial bone marrow aspirates are shown as a bar chart; the black areas and white areas indicate the numbers of tumor cells and other cells, respectively. The change in skin lesions is also illustrated. Hematological data are shown as line charts. ASR, atraumatic splenic rupture; WBC, white blood cell; Hb, hemoglobin; Plt, platelet.

Cardiopulmonary resuscitation was not attempted following the previously obtained informed consent. The clinical course is summarized in Figure 5. Marked hemoperitoneum, and the progression of splenomegaly and lymphadenopathy were found on autopsy imaging. Furthermore, autopsy revealed $1550 \mathrm{~mL}$ of hemorrhagic ascites and splenomegaly with capsule rupture and hematoma, indicating that ASR was the source of the marked hemorrhage. On microscopic analysis, bone marrow and lymph nodes were packed with tumor cells, demonstrating progression of the disease (Figure 6A, 

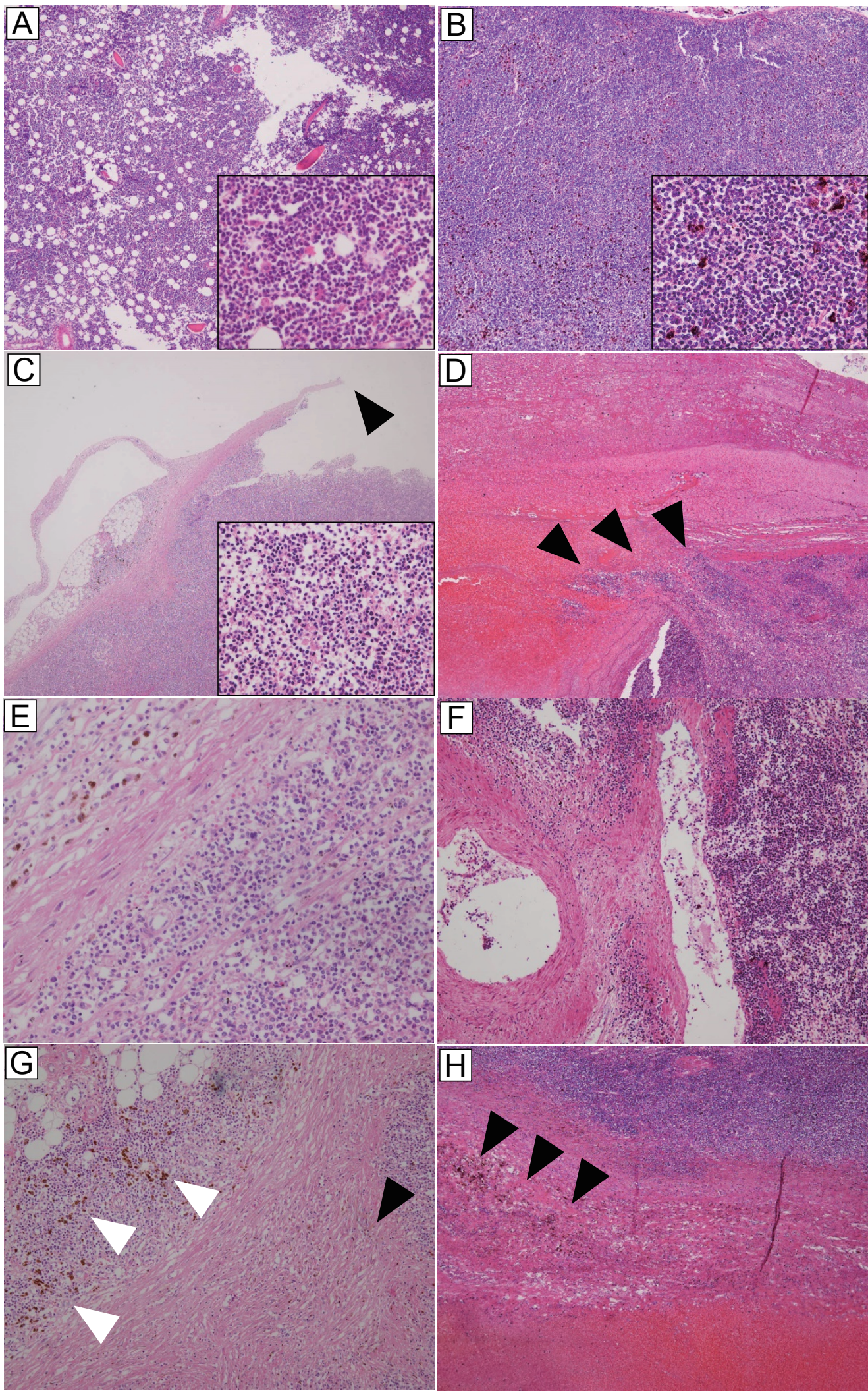

Fig. 6. Autopsy specimens. $(\boldsymbol{A})$ The bone marrow was packed with tumor cells. The high-power field is shown at the bottom-right. $(\boldsymbol{B})$ The mediastinal lymph node was packed with tumor cells. The high-power field is shown at the bottom-right. $(\boldsymbol{C})$ The spleen was packed with tumor cells. The black triangle indicates disruption of the splenic capsule. The parenchyma of the spleen was also disrupted at the same position. The high-power field is shown at the bottom-right. $(\boldsymbol{D})$ The hematoma was directly attached to the parenchyma of the spleen (black triangles), indicating disappearance of the splenic capsule. $(\boldsymbol{E})$ The splenic capsule demonstrated thinning and was infiltrated by tumor cells. $(\boldsymbol{F})$ The vessel walls were also infiltrated by tumor cells. $(\boldsymbol{G})$ The black triangle indicates the fibrotic tissue and white triangles indicate macrophages engulfing hemosiderin at the splenic capsule. These findings are consistent with prior ASR. $(\boldsymbol{H})$ Black triangles indicate macrophages engulfing hemosiderin near the hematoma, suggesting that the prior ASR occurred at the same position. ASR, atraumatic splenic rupture. 
Table 1. The number of cases of ASR associated with hematological malignancies reported between 1994 and 2018. The cases were extracted from 2 review articles and online sources. ASR, atraumatic splenic rupture.

\begin{tabular}{|c|c|c|}
\hline Disease & $\begin{array}{l}\text { Number } \\
\text { of cases }\end{array}$ & References \\
\hline $\begin{array}{l}\text { Non-Hodgkin's } \\
\text { lymphoma }\end{array}$ & 26 & $\begin{array}{l}(1,2) \\
\text { Oinonen et al. Ann Hematol. (1997) } \\
\text { Biswas et al. World J Emerg Surg. } \\
(2006) \\
\text { Dayama et al. Mediterr J Hematol } \\
\text { Infect Dis. (2011) } \\
\text { Tan et al. Case Rep Med. (2012) } \\
\text { Mohammed et al. J Surg Case Rep. } \\
\text { (2016) } \\
\text { Komeno et al. Intern Med. (2017) }\end{array}$ \\
\hline $\begin{array}{l}\text { Acute lymphoblastic } \\
\text { leukemia }\end{array}$ & 9 & $(1,2)$ \\
\hline $\begin{array}{l}\text { Acute myelogenous } \\
\text { leukemia }\end{array}$ & 8 & $\begin{array}{l}(1,2) \\
\text { Kuba } \text { et al. Biomed Pap Med Fac Univ } \\
\text { Palacky Olomouc Czech Repub. (2015) } \\
\text { Zeidan et al. Leuk Lymphoma. (2014) } \\
\text { De Santis et al. Rev Bras Hematol } \\
\text { Hemoter. (2014) } \\
\text { Zhang et al. Mol Clin Oncol. (2017) }\end{array}$ \\
\hline $\begin{array}{l}\text { Blastic plasmacytoid } \\
\text { dendritic cell } \\
\text { neoplasm }\end{array}$ & 5 & $\begin{array}{l}\text { Chee et al. Br J Haematol. (2006) } \\
\text { Kawashima et al. Rinsho Ketsueki. } \\
\text { (2013) } \\
\text { Köpeczi et al. Rev Romana Med Lab. } \\
\text { (2014) } \\
\text { Tenreiro et al. J Gastrointest Surg. } \\
\text { (2018) } \\
\text { and present case }\end{array}$ \\
\hline Hairy cell leukemia & 3 & $\begin{array}{l}\text { (2) } \\
\text { Gedik et al. World J Gastroenterol. } \\
\text { (2008) }\end{array}$ \\
\hline $\begin{array}{l}\text { Chronic myelogenous } \\
\text { leukemia }\end{array}$ & 2 & $\begin{array}{l}\text { (2) } \\
\text { Galhotra et al. Indian J Surg Oncol. } \\
\text { (2011) }\end{array}$ \\
\hline Multiple myeloma & 2 & $\begin{array}{l}\text { (2) } \\
\text { Ho et al. Clin Med Case Rep. (2008) }\end{array}$ \\
\hline $\begin{array}{l}\text { Chronic lymphocytic } \\
\text { leukemia }\end{array}$ & 1 & (1) \\
\hline $\begin{array}{l}\text { Adult T-cell leukemia/ } \\
\text { lymphoma }\end{array}$ & 1 & (2) \\
\hline
\end{tabular}

B). However, morphological transformation was not observed. The spleen was also packed with tumor cells, and disruption of the splenic capsule and adherence of hematoma were noted (Figure 6C, D). The splenic capsule exhibited thinning and was infiltrated by tumor cells (Figure 6E). The vessel walls were also disrupted due to tumor cell infiltration (Figure 6F). These findings suggested that the disruptive infiltration of tumor cells into the splenic capsule and vessel walls lead to ASR and marked hemorrhage. In addition, the spleen was adhered to the small intestine and diaphragm, and fresh and old hematoma contained fibrotic tissue and macrophages engulfing hemosiderin (Figure 6G, H). This was indicative of repeated ASRs. Tumor cells were also found in the liver, lungs, kidneys, adrenal glands and epicardium. Immunohistochemical examination revealed the same immunophenotype of the tumor cells as at diagnosis.
ASR is a rare complication of hematological neoplasms, including BPDCN. ${ }^{1,2}$ In the present case, the patient with BPDCN experienced three ASRs. The first two were mild ruptures and remission occurred, whereas the last one was severe and fatal. This patient's course provided two important clinical suggestions.

First, ASR can cause spontaneous partial remission in patients with BPDCN. Previously, 2 BPDCN cases of spontaneous remission were reported, but the triggers and mechanisms were not elucidated. ${ }^{3,4}$ Our patient exhibited spontaneous partial remission after mild ASR. This is the first report describing spontaneous partial remission after ASR. As the mechanism of this phenomenon, we hypothesized that the dissemination of tumor cells in the peritoneal cavity caused some immune reaction resembling the immunotherapy using autologous cells. Recently, a number of cancer vaccinations administering autologous tumor cells are being explored for different neoplasms, and the induction of tumor reactive $\mathrm{T}$ cells, and therapeutic efficacy in mouse models and clinical practice were reported..$^{5-13}$ The present patient exhibited high grade fever and elevated CRP and uric acid levels after the first and the second ASRs. These findings suggested that splenic rupture with the dissemination of tumor cells in the peritoneal cavity evoked some immune reaction that resulted in the systemic eradication of tumor cells. Previous reports describing cancer vaccination demonstrated that autologous tumor cells effectively induce antitumor immunity after being engineered to express granulocyte macrophage colony-stimulating factor, ${ }^{5-8}$ interleukin $2^{6,9}$ interferon gamma ${ }^{6}$ or CD40 ligand. ${ }^{9}$ These molecules are known to be produced by activated lymphocytes under inflammatory conditions. Thus, we hypothesized that autologous tumor cells disseminated by splenic rupture played a role similar to that of cancer vaccination in the present case.

Second, ASR may be a relatively frequent complication in patients with BPDCN. In rare instances, ASR occurs in variety of hematological neoplasms. As the disease entity of BPDCN was established in 1994, we extracted and calculated the number of cases of ASR associated with hematological malignancies reported between 1994 and 2018 from 2 review articles $^{1,2}$ and online sources; Case reports were searched on PubMed or Google scholar using the following terms: "splenic rupture leukemia", "splenic rupture lymphoma", "splenic rupture myeloma" or "splenic rupture blastic plasmacytoid dendritic cell neoplasm". The total number of cases of each hematological neoplasm is listed in Table 1. Cases of non-Hodgkin's lymphoma were the most common, followed by ALL, AML and BPDCN. We must note that our evaluation method contains a publication bias and the incidence of rare diseases may be overestimated. However, the prevalence of BPDCN was much lower than that of the other listed hematological diseases; therefore, it is possible that the incidence of ASR is higher in patients with BPDCN. All five BPDCN cases in Table 1 had accompanying splenomegaly, although this is not a common finding in patients with BPDCN. In addition, ASR occurred at diagnosis in 3 cases, at relapse in one case and during the refractory phase in our 
case. These findings suggest that ASR is associated with splenomegaly and disease progression. Autopsy suggested that the disruptive infiltration of tumor cells into the splenic capsule and vessel walls lead to ASR. This infiltration capability may be the cause of the high incidence of ASR. Previously, age above 40, splenomegaly and associated malignancy were reported as risk factors for ASR-related mortality. ${ }^{14}$ Our patient had all these factors, indicative of a markedly high risk. Thus, we should be aware of the possibility of ASR in elderly patients with BPDCN and splenomegaly, especially at the time of disease progression.

In conclusion, we reported the first case of BPDCN in which spontaneous partial remission was achieved after ASR. Further accumulation of clinical evidence is needed to elucidate the mechanism of this phenomenon. However, fatal ASR should be prevented in order to avoid the sudden death of elderly patients with BPDCN and splenomegaly.

\section{CONFLICT OF INTEREST}

The authors declare that they have no conflict of interest.

\section{REFERENCES}

1 Giagounidis AA, Burk M, Meckenstock G, Koch AJ, Schneider W. Pathologic rupture of the spleen in hematologic malignancies: two additional cases. Ann Hematol. 1996; 73 : 297-302.

2 Aubrey-Bassler FK, Sowers N. 613 cases of splenic rupture without risk factors or previously diagnosed disease: a systematic review. BMC Emerg Med. 2012; 12 : 11.

3 Hashikawa K, Niino D, Yasumoto S, et al. Clinicopathological features and prognostic significance of CXCL12 in blastic plasmacytoid dendritic cell neoplasm. J Am Acad Dermatol. 2012; $66: 278-291$.

4 Yasuda H, Takaku T, Tomomatsu J, et al. Spontaneous regression of cutaneous blastic plasmacytoid dendritic cell neoplasm followed by acute monocytic leukemia evolving from myelodysplastic syndrome. Intern Med. 2014; 53 : 2717-2720.

5 Dranoff G, Jaffee E, Lazenby A, et al. Vaccination with irradiated tumor cells engineered to secrete murine granulocyte-macrophage colony-stimulating factor stimulates potent, specific, and long-lasting anti-tumor immunity. Proc Natl Acad Sci USA. 1993; $90: 3539-3543$.

6 Maini A, Nishisaka N, Kinoshita Y, et al. Combination of radiation and vaccination with autologous tumor cells expressing IL-2, IFN-gamma and GM-CSF for treatment of murine renal carcinoma. In Vivo. 2003; 17 : 119-123.

7 Luiten RM, Kueter EW, Mooi W, et al. Immunogenicity, including vitiligo, and feasibility of vaccination with autologous GM-CSF-transduced tumor cells in metastatic melanoma patients. J Clin Oncol. 2005; $23:$ 8978-8991.
8 Ho VT, Vanneman M, Kim H, et al. Biologic activity of irradiated, autologous, GM-CSF-secreting leukemia cell vaccines early after allogeneic stem cell transplantation. Proc Natl Acad Sci USA. 2009; $106: 15825-15830$.

9 Okur FV, Yvon E, Biagi E, et al. Comparison of two CD40ligand/interleukin-2 vaccines in patients with chronic lymphocytic leukemia. Cytotherapy. 2011; 13 : 1128-1139.

10 Burkhardt UE, Hainz U, Stevenson K, et al. Autologous CLL cell vaccination early after transplant induces leukemia-specific T cells. J Clin Invest. 2013; $123: 3756-3765$.

11 Gibbins JD, Ancelet LR, Weinkove R, et al. An autologous leukemia cell vaccine prevents murine acute leukemia relapse after cytarabine treatment. Blood. 2014; 124 : 2953-2963.

12 Curry WT Jr, Gorrepati R, Piesche M, et al. Vaccination with irradiated autologous tumor cells mixed with irradiated GM-K562 cells stimulates antitumor immunity and T lymphocyte activation in patients with recurrent malignant glioma. Clin Cancer Res. 2016; 22 : 2885-2896.

13 Ho VT, Kim HT, Bavli N, et al. Vaccination with autologous myeloblasts admixed with GM-K562 cells in patients with advanced MDS or AML after allogeneic HSCT. Blood Adv. 2017; $1: 2269-2279$.

14 Renzulli P, Hostettler A, Schoepfer AM, Gloor B, Candinas D. Systematic review of atraumatic splenic rupture. Br J Surg. 2009; 96 : 1114-1121.

Shinya Daitoku, ${ }^{1,2)}$ Mitsuho Onimaru, ${ }^{3}$ Kazuki Tanimoto ${ }^{2)}$ and Mika Kuroiwa ${ }^{1)}$

${ }^{1)}$ Department of Hematology, National Hospital Organization Fukuoka Higashi Medical Center, Koga, Japan, ${ }^{2}$ Department of Hematology and Oncology, Japanese Red Cross Fukuoka Hospital, Fukuoka, Japan, ${ }^{3}$ Division of Pathophysiological and Experimental Pathology, Department of Pathology, Graduate School of Medical Science, Kyushu University, Fukuoka, Japan

Corresponding author: Shinya Daitoku, M.D., Ph.D., Department of Hematology and Oncology, Japanese Red Cross Fukuoka Hospital, 3-1-1 Ohkusu Minami-ku Fukuokashi, Fukuoka 815-8555, Japan. E-mail: daitokuji.nattou@gmail.com

Received: May 21, 2018.

Revised: November 27, 2018.

Accepted: December 19, 2018.

Online Published: March 27, 2019

DOI:10.3960/jslrt.18021

Copyright $\odot 2018$ The Japanese Society for Lymphoreticular Tissue Research (c) BY-NC-SA This work is licensed under a Creative Commons AttributionNonCommercial-ShareAlike 4.0 International License. 